\title{
A statistical protocol for a holistic adjustment of global sea level budget
}

DOI: https://doi.org/10.1515/jogs-2020-0001

Received July 10, 2019; accepted November 27, 2019

\begin{abstract}
Current studies in global mean sea level, GMSL, studies assess the closure/misclosure of the GMSL budget components and their uncertainties. Because Earth's hydrosphere conserves water, a closed global mean sea level budget with a consistent set of estimates and their statistics is necessary. An unclosed budget means that there are problems to be addressed such as biases in the budget components, unreliable error statistics about the estimates, unknown or known but unmodeled budget components. In a misclosed global mean sea level budget, as practiced in recent studies, the trend estimates for the budget components and their errors account only for the anomalies of each budget component in isolation. On the other hand, the trend of each series must consider the trends of the other series in tandem such that the global mean sea level budget is closed for a holistic assessment, which can only be achieved by adjusting global mean sea level budget components simultaneously. In this study, we demonstrate a statistical protocol to ameliorate this deficiency, which potentially have implications for future sea level science studies, including the future Intergovernmental Panel on Climate Change (IPCC) Assessment Reports, and the US Climate Assessment Reports.
\end{abstract}

Keywords: Global sea level budget; misclosure; Condition equations; Satellite altimetry; Climate change

\section{Introduction}

It is imperative to better understand and improve the current global mean sea level, (GMSL) budget with a consistent set of estimates for its components, with the goal to differentiate anthropogenic contributions to the climate

\footnotetext{
*Corresponding Author: H. Bâki Iz: Division of Geodetic Science, School of Earth Sciences, The Ohio State University, Columbus, Ohio, USA, E-mail: H.Baki.Iz@gmail.com

Visiting scholar

C. K. Shum: Division of Geodetic Science, School of Earth Sciences, The Ohio State University, Columbus, Ohio, USA, and Institute of Geodesy \& Geophysics, Chinese Academy of Sciences, Wuhan, Hubei 430077, China
}

change from natural variations and to enable more accurate prediction/projection of GMSL rise.

Ocean thermal expansion and halosteric contraction, ice sheet, mountain glacier and ice cap mass balance, changes in terrestrial and surface and ground water storage, anthropogenic impoundment of waters in dams, and to a much lesser importance, permafrost degradation, snow and atmospheric water vapor change, are all identified as major components of geophysical contributions to the present-day global mean sea-level variations. Recent report by the World Climate Research Program, WCRP's Global Sea Level Budget Group elaborates exhaustively on the GMSL budget for the period 1993-present and its components using recent literature on sea level science including the US Fourth Climate Assessment Report, Vol. 1, 2017, and the latest Intergovernmental Panel on Climate Change (IPCC) Fifth Assessment Report (IPCC, 2014).

Current practice of evaluating GMSL budget is to quantify the linear rate of change of its geophysical contributions (components) from various sources, and to calculate the misclosure of the budget (deviation from zero) together with the error of the misclosure (WCRP, 2018). In this study, we briefly discuss that this is an interim result, which may lead to ambiguous outcomes. We then propose and demonstrate a statistical protocol to ameliorate this ambiguity by adjusting previously estimated trends ${ }^{1}$ of the budget components and their uncertainties within a period with a GMSL budget closure constraint. The readjustment of the velocities of the budget components does not mean that the underlying sources of errors leading to the misclosure were eliminated, but its components and their uncertainties are reassessed under the GMSL budget closure constraint.

\section{GMSL budget velocities and GMSL budget misclosure}

We will use independently estimated velocities during 2005 - to present reported by WCRP (2018) listed in their

1 Trend, velocity and rate are used interchangeably in this study. 
Table 13, which are listed in the following Table. WCRP (2018) study exhaustively covers information about the GMSL budget components enabling an assessment of the GMSL budget closure/misclosure ${ }^{2}$ for one of its scenarios, which is $0.55 \pm 0.50 \mathrm{~mm} / \mathrm{yr}$ for the period 2005 - to present. The certitude of the GMSL budget relies heavily on this number and its error. Nonetheless, this is the misclosure of a most recently assessed GMSL budget, which is ambiguous hence uninformative. The magnitude of the misclosure is so large that another unknown budget component as large as Antarctic may missing in the GMSL budget. Yet, the root sum square, RSS, uncertainty of the misclosure has a large borderline value that one cannot confidently reject or accept the null hypothesis (the GMSL budget is closed) for the GMSL budget closure. This dilemma occurs because the quantified misclosure is not the end but an interim value, which is not appropriate for a final assessment. Note that we can make the same argument for the GSML assessment for the period 1993 - present displayed in Table 12 of WCRP, (2018) or any other assessments to be made in the near future. In the following section, we will establish an optimal statistical protocol to remove this ambiguity.

\section{Equation for Global Mean Sea Level Budget}

Following the narrative in WCRP (2018), Earth's hydrosphere conserves water, i.e.,

$$
\begin{aligned}
& M_{\text {OCEAN }}+M_{\text {GLACIERS }}+M_{\text {GREENLAND }}+M_{\text {ANTARCTICA }} \\
& +M_{T W S}+M_{\text {SNOW }}+M_{W V}+M_{\text {PERMAFROST }}=\mathrm{constant}
\end{aligned}
$$

where the components of the above expression represent mass of oceans, glaciers, Greenland and Antarctica ice sheets, terrestrial water storage, snow, atmospheric water vapor, and permafrost respectively. Hence, the sum of the time evolution of the components, denoted by $M(t)$, can be represented as,

$$
\begin{aligned}
& M(t)_{\text {OCEAN }}+M(t)_{\text {GLACIERS }}+M(t)_{\text {GREENLAND }} \\
& +M(t)_{\text {ANTARCTICA }}+M(t)_{\text {TWS }}+M(t)_{\text {SNOW }}+M(t)_{W V} \\
& +M(t)_{\text {PERMAFROST }}=0
\end{aligned}
$$

2 The reported value for the misclosure $0.55 \pm 0.3 \mathrm{~mm} / \mathrm{yr}$ by the WGRP (2018) in its Table 13 is computationally erroneous. It should be 0.55 $\pm 0.50 \mathrm{~mm} / \mathrm{yr}$ (Root Sum Square RSS error of the misclosure cannot be smaller than the standard error of any one of its components, such as the standard error of the thermosteric component, which is 0.40 $\mathrm{mm} / \mathrm{yr}$ ). Similar error also exists for the misclosure evaluated for the period 1993-present in their Table 12.
These changes are part of the GMSL anomalies, $G M S L(t)$, which can be expressed as a function of change in steric and ocean mass as a lump-sum,

$$
\begin{aligned}
& \operatorname{GMSL}(t)=G M S L(t)_{\text {THERMOSTERIC }}+G M S L(t)_{\text {HALOSTERIC }} \\
& +G M S L(t)_{\text {OCEANMASS }}
\end{aligned}
$$

where $G M S L(t)_{\text {THERMOSTERIC }}$ and GMSL $(t)_{\text {HALOSTERIC }}$ components refer to the contributions of ocean thermal expansion and salinity to sea-level change (steric) respectively. GMSL $(t)_{O C E A N M A S S}$, equivalently $M(t)_{\text {OCEANMASS }}$, refers to the change in mass of oceans, which can be inferred from Eq. (2). Therefore, in light of Eq. (2) and Eq. (3), GMSL budget equation is represented as,

$$
\begin{aligned}
& G M S L(t)-G M S L_{\text {THERMOSTERIC }}-G M S L_{\text {HALOSTERIC }} \\
& -M(t)_{\text {OCEAN }}-M(t)_{\text {GLACIERS }}-M(t)_{\text {GREENLAND }} \\
& -M(t)_{\text {ANTARCTICA }}-M(t)_{\text {TWS }}-M(t)_{\text {SNOW }}-M(t)_{W V} \\
& -M(t)_{\text {PERMAFROST }}=0
\end{aligned}
$$

This expression is an idealized representation and because each component is inferred from observations that are subject to errors, the operational budget equation is not closed (misclosure). The following statistical protocol will adjust the operationally realized GMSL budget components (geophysical contributions to GMSL) using the method of condition equations.

\section{Method of condition equations and its application to adjust the GMSL budget}

The method of condition equation, also known as method of correlation, or method of correlates, or equation of condition, is used frequently in geodetic practice. Its first use as an "equation of condition" goes back to Johann Tobias Mayer (1723-1762), a cartographer and practical astronomer, followed by Helmert Friedrich Robert (1843-1917), a geodesist who formalized and extended the method to include unknown parameters. In this note, we choose not to include its derivation for brevity, but followed the outcome of the derivations and notations used in Uotila (1997) with minor modifications and simplifications. The complete derivation of the method is lengthy but available through the online link to the book by Wells and Krakiwsky, (1971) listed in the references.

For the numerical example, we will use the data from one of the scenarios extracted from the Table 12 of WCRP (2018), which is listed here in the second column of Table 1. 
Table 1. Independently estimated and adjusted GMSL budget components. The remaining last digit in the adjusted misclosure, $0.01 \mathrm{~mm}$, is the round-off error.

\begin{tabular}{llcc}
\hline No & GMSL Budget Component & $\begin{array}{c}\text { Independently Estimated trend (mm/yr) } \\
\text { 2005- to present (WCRP, 2018) }\end{array}$ & $\begin{array}{l}\text { Adjusted trend } \\
\text { (mm/yr) }\end{array}$ \\
\hline 1 & GMSL & $3.50 \pm 0.20$ & $3.46 \pm 0.14$ \\
2 & Thermosteric sea level (full depth) & $1.30 \pm 0.40$ & $1.31 \pm 0.07$ \\
3 & Glaciers & $0.74 \pm 0.10$ & $0.89 \pm 0.24$ \\
4 & Greenland & $0.76 \pm 0.10$ & $0.91 \pm 0.24$ \\
6 & Antarctica & $0.42 \pm 0.10$ & $0.57 \pm 0.24$ \\
6 & TWS from GRACE & $-0.27 \pm 0.15$ & $-0.20 \pm 0.18$ \\
& Misclosure = GMSL minus & & 0.01 \\
7 & sum of the GMSL budget & $0.55 \pm 0.50$ & \\
& components & & \\
\hline
\end{tabular}

Under this scenario, the atmospheric water vapor, snow, and permafrost contributions are ignored as being small to conform with the synthesis given in WCRP (2018). Only the 2005-present time period covered by Argo data and GRACE gravimetry observation is considered. The corresponding GMSL budget equation is now stated as,

$$
\begin{aligned}
& l_{\text {GMSL }}-l_{\text {THERMOSTERIC }}-l_{\text {GLACIERS }}-l_{\text {GREENLAND }} \\
& -l_{\text {ANTARCTIC }}-l_{\text {TWS }}=0
\end{aligned}
$$

Where $l$ represents the linear rate of change for each component estimated from their corresponding time series with their errors estimates. These estimates are to be treated as proxy observations for the following adjustment. The above GMSL budget expression states a theoretical, idealized closure of the geophysical contributors of present-day global sea level rise. Because each component is inferred from a time series subject to error, the operational GMSL budget equation is,

$$
\begin{aligned}
& \hat{l}_{\text {GMSL }}-\hat{l}_{\text {THERMOSTERIC }}-\hat{l}_{\text {GLACIERS }}-\hat{l}_{\text {GREENLAND }} \\
& -\hat{l}_{\text {ANTARCTIC }}-\hat{l}_{\text {TWS }}=0
\end{aligned}
$$

where, $\hat{l}=l^{b}+v$ is the sum of the observed contributors (estimate independently adjusted using their time series) $l^{b}$, and the realization of their true errors $v$ as residuals, which are to be adjusted such that the budget equation is closed. With this understanding, the condition equation for an operationally realized GMSL budget is written as,

$$
\begin{aligned}
& v_{\text {GMSL }}-v_{\text {THERMOSTERIC }}-v_{\text {GLACIERS }}-v_{\text {GREENLAND }} \\
& -v_{\text {ANTARCTIC }}-v_{\text {TWS }}+\left(l_{\text {GMSL }}^{b}-l_{\text {THERMOSTERIC }}^{b}-l_{\text {TNTARCTIC }}^{b}-l_{\text {TWS }}^{b}\right)=0 \\
& -l_{\text {GLACIERS }}^{b}-l_{\text {GREENLAND }}^{b}-l_{\text {ANT }}^{b}
\end{aligned}
$$

This equation can be expressed in matrix notation as,

$$
\underset{1 \times 6}{B} \underset{6 \times 1}{V}+\underset{1 \times 1}{W}=0
$$

where, $B$ is the $1 \times 6$ coefficient vector (it would have been a matrix if there were more than a single condition),

$$
B=\left[\begin{array}{llllll}
1 & -1 & -1 & -1 & -1 & -1
\end{array}\right]
$$

and $V^{T}$ is the vector of residuals representing,

$$
\begin{aligned}
& V^{T}:=\left[v_{\text {GMSL }}-v_{\text {THERMOSTERIC }}-v_{\text {GLACIERS }}-v_{\text {GREENLAND }}\right. \\
& \left.-v_{\text {ANTARCTIC }}-v_{T W S}\right]
\end{aligned}
$$

Because previously and independently estimated linear trends for each GMSL budget component are considered as observations, they are subject to errors. Hence, the GMLS budget cannot be closed. This misclosure, $W$, can be computed using the data given in Table 1 ,

$$
\begin{aligned}
& W:=\left[l_{\text {GMSL }}^{b}-l_{\text {THERMOSTERIC }}^{b}-l_{\text {GLACIERS }}^{b}-l_{\text {GREENLAND }}^{b}\right. \\
& \left.-l_{\text {ANTARCTIC }}^{b} l_{\text {TWS }}^{b}\right]=0.55 \mathrm{~mm} / \mathrm{yr}
\end{aligned}
$$

The variance-covariance, $\mathrm{V} / \mathrm{C}$, matrix $\sum_{L^{b}}$ of the observed GMSL budget components is given by,

$$
\sum_{L^{b}}=\sigma_{0}^{2} P^{-1}
$$

where $P$ is the $6 \times 6$ weight matrix in this example. By definition, weights are given by $p_{i}:=\frac{\sigma_{0}^{2}}{\sigma_{i}^{2}}$ where $\sigma_{i}^{2}$ is the variance of the $i^{t h}$ budget component. Adopting the a priori variance of unit weight $\sigma_{0}^{2}=1$, the V/C matrix of the GMSL budget component is,

\section{$\sum_{L^{b}}=$}

$\left[\begin{array}{cccccc}\sigma_{\text {GMSL }}^{2} & 0 & 0 & 0 & 0 & 0 \\ 0 & \sigma_{\text {THERMO }}^{2} & 0 & 0 & 0 & 0 \\ 0 & 0 & \sigma_{\text {GLACIERS }}^{2} & 0 & 0 & 0 \\ 0 & 0 & 0 & \sigma_{\text {GREENLAND }}^{2} & 0 & 0 \\ 0 & 0 & 0 & 0 & \sigma_{\text {ANTARCTIC }}^{2} & 0 \\ 0 & 0 & 0 & 0 & 0 & \sigma_{\text {TWS }}^{2}\end{array}\right]$

The above V/C matrix can be quantified using the standard errors of the independently estimated linear rates for each budget component listed in Table 1 . 


$$
\sum_{L^{b}}=\left[\begin{array}{cccccc}
0.20^{2} & 0 & 0 & 0 & 0 & 0 \\
0 & 0.40^{2} & 0 & 0 & 0 & 0 \\
0 & 0 & 0.10^{2} & 0 & 0 & 0 \\
0 & 0 & 0 & 0.10^{2} & 0 & 0 \\
0 & 0 & 0 & 0 & 0.10^{2} & 0 \\
0 & 0 & 0 & 0 & 0 & 0.15^{2}
\end{array}\right]
$$

Note that the GMSL budget components are physically correlated. Potential lagged cross-correlations among the budget components' errors are therefore important for proper assessment of the uncertainties. At this point, we do not have enough information to quantify a full $\mathrm{V} / \mathrm{C}$ covariance matrix. Nonetheless, this setup can also accommodate covariances among the GMSL budget uncertainties as they become available.

Expressing the relationship between the vector of adjusted values of observed quantities $\hat{L}$,

$$
\hat{L}^{b}:=\left[\begin{array}{llll}
\hat{l}_{G M S L}^{b} & \hat{l}_{\text {THERMOSTERIC }}^{b} \hat{l}_{\text {GLACIERS }}^{b} \hat{l}_{\text {GREENLAND }}^{b} \hat{l}_{\text {ANTARCTIC }}^{b} \hat{l}_{T W S}^{b}
\end{array}\right]
$$

and their vector of observed realizations $L^{b}$ (independently estimated trends) as,

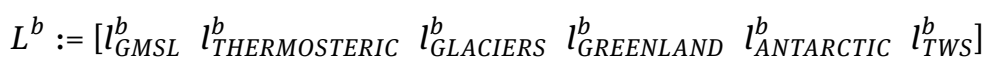

the corresponding vector of residuals $V$ is stated as follows,

$$
\hat{L}=L^{b}+V \text { and } V=\hat{L}-L^{b}
$$

We now impose the following condition for adjusting the GMSL budget components to close,

$$
B \hat{L}=0 \quad \text { or equivalently } \mathrm{B}\left(L^{b}+V\right)=0
$$

This equation admits infinitely many solutions. Among them the one which fulfills $V^{T} P V=\min$ is to be selected. Hence, the combined target function to be minimized subject to $B V+W=0$ can be formulated using Lagrange multipliers, $K$, as follows,

$$
\begin{aligned}
& V^{T} P V-2 K^{T}(B V+W)=\min \\
& \text { s.t. } \\
& B V+W=0
\end{aligned}
$$

Taking the appropriate derivatives with respect to $K$ and $V$ and setting them to zero, we obtain,

$$
\begin{aligned}
& V=-P^{-1} B^{T}\left(B P^{-1} B^{T}\right)^{-1} W \\
& V^{T}=\left[\begin{array}{llllll}
-0.037 & 0.009 & 0.146 & 0.146 & 0.146 & 0.065
\end{array}\right]
\end{aligned}
$$

The adjusted observation vector $\hat{L}$ is now quantified as,

$$
\begin{aligned}
& \hat{L}=L^{b}+V \\
& \hat{L}^{T}=\left[\begin{array}{llllll}
3.46 & 1.31 & 0.89 & 0.91 & 0.57 & -0.20
\end{array}\right]
\end{aligned}
$$

and as a check, given $B$ and $\hat{L}, B \hat{L}=0$ is numerically verified.

The a posteriori variance of unit weight $\hat{\sigma}_{0}$ is quantified as,

$$
\hat{\sigma}_{0}=\sqrt{\frac{V^{T} P V}{r}}=0.028
$$

where $r$ is the number of condition equations which is 1 in this example.

Applying variance propagation to Eq. (22), the following V/C matrix of the adjusted GMSL budget components is obtained,

$$
\hat{L}=L^{b}+V \rightarrow \sum_{L}=\sum_{L^{b}}-\sum_{V}
$$


This expression can be expanded to quantify,

$$
\begin{aligned}
\sum_{\hat{L}} & =\sigma_{o}^{2}\left(P^{-1}-P^{-1} B^{T}\left(B P^{-1} B^{T}\right)^{-1} B P^{-1}\right) \\
\sum_{\hat{L}} & =\left[\begin{array}{ccccccc}
0.0188 & 0.0003 & 0.0054 & 0.0054 & 0.0054 & 0.0024 \\
0.0003 & 0.0049 & -0.0013 & -0.0013 & -0.0013 & -0.0006 \\
0.0054 & -0.0013 & 0.0591 & -0.0214 & -0.0214 & -0.0095 \\
0.0054 & -0.0013 & -0.0214 & 0.0591 & -0.0214 & -0.0095 \\
0.0054 & -0.0013 & -0.0214 & -0.0214 & 0.0591 & -0.0095 \\
0.0024 & -0.0006 & -0.0095 & -0.0095 & -0.0095 & 0.0316
\end{array}\right]
\end{aligned}
$$

The corresponding standard errors of the adjusted velocities of the GMSL budget components are calculated through the diagonal elements of the above $\mathrm{V} / \mathrm{C}$ matrix,

$$
\sigma=\left[\begin{array}{llllll}
0.137 & 0.070 & 0.243 & 0.243 & 0.243 & 0.178
\end{array}\right]
$$

The correlations among the velocities of the GMSL budget components can also be assessed directly from $\sum_{\hat{L}}$,

$$
\sum_{\hat{L}}^{\text {Correlation }}=\left[\begin{array}{cccccc}
1 & 0.03 & 0.16 & 0.16 & 0.16 & 0.1 \\
0.03 & 1 & -0.08 & -0.08 & -0.08 & -0.05 \\
0.16 & -0.08 & 1 & -0.36 & -0.36 & -0.22 \\
0.16 & -0.08 & -0.36 & 1 & -0.36 & -0.22 \\
0.16 & -0.08 & -0.36 & -0.36 & 1 & -0.22 \\
0.1 & -0.05 & -0.22 & -0.22 & -0.22 & 1
\end{array}\right]
$$

The results suggest that the correlations among the trends of the GMSL budget components are small but still require further verification because of the limited resolution in representing the variations in the GMSL budget components using trends (velocities) rather than the actual series.

The final tally of the adjusted budget components is listed in Table 1. The solution reveals that the adjusted trends do not deviate significantly from their unadjusted trends. All GMSL budget trends together fulfill the GMSL budget constraint. Most importantly, the ambiguity of the misclosure is no longer an impediment for the assessment of the GMSL budget as a whole and its components individually in relation to each other. However, note that this study is by no means exhaustive and no strong claims can be made for the numerical results in confidence because the input velocities were only intended to demonstrate the statistical protocol. The admissibility of the adjusted trends is therefore left to the experts of each discipline of each budget component.

Nonetheless, this example in its limited scope reveals that because the adjusted values are not significantly different from their originals in magnitude, only small improvements are required for each budget component in the near future $^{3}$. As the accuracy and precision of the series for the series improve with the increasing length of the series, the same protocol can be used repeatedly to obtain updated and improved estimates for trends for all GMSL budget components. These improvements will also increase the resolution and thereby enable the introduction of new series, such as atmospheric water vapor and permafrost melting, which were omitted as being negligible in this example.

3 As one of the reviewers commented "It could be that there are significant counterbalancing errors in the original estimates requiring more substantial revisions to properly close the sea level budget.” That is correct. Nonetheless, one must have at least two budget components going wrong at the same time and in opposite directions, which is likely to be detected beforehand. 


\section{Conclusion}

In this contribution, quantification of GMSL misclosure and its uncertainties, as commonly used by recent GMSL budget studies, is found to be ambiguous partly because the estimated misclosure is large, its error is discouragingly wide and cannot be tested statistically. However, if we frame this inference in the context of the proposed statistical protocol, the closure for the GMSL budget can be achieved by readjusting the GMSL budget component trends and their standard errors by small amounts. This assessment is not possible if the analysis stops at the misclosure stage, as in recent studies. The new adjusted values and their error estimates for the GMSL budget components fulfill the requirement for the GMSL budget to close. We would like to emphasize again that closing the GMSL budget does not mean that the misclosure is ignored but simply accounted for each budget component in a least squares sense.

In this study we intended to introduce a century long well-known adjustment method in geodetic science known as "Condition Equations" to the sea level studies. It is possible to extend this model using observed time series of the GMSL budget components (monthly or yearly instead of velocities (trends) as observations while estimating the velocities of the budget components simultaneously without readjusting them. Such formulation will enable closing the GMSL budget at epoch by epoch and the topic of a follow up study.
Acknowledgments: We gratefully acknowledge two reviewers for their helpful comments.

\section{References}

Helmert, F. R.,1872, Ausgleichungsrechnung nach der Methode der kleinsten Quadrate, mit Anwendungen auf die Geodäsie und die Theorie der Messinstrumente.

IPCC, 2014, Climate Change, 2014, Synthesis Report. Contribution of Working Groups I, II and III to the Fifth Assessment Report of the Intergovernmental Panel on Climate Change [Core Writing Team, R.K. Pachauri and L.A. Meyer (eds.)]. IPCC, Geneva, Switzerland, $151 \mathrm{pp}$.

Mayer, T., in Stigler, S. M., 1986, The History of Statistics: The Measurement of Uncertainty before 1900. Cambridge, Mass.: Belknap Press of Harvard University Press.

Uotila, U., 1988, Analysis of Observations. Lecture Notes, The Ohio State University.

US Fourth Climate Assessment Report, Vol. 1, 2017, https://science2017.globalchange.gov, accessed, Feb 2019.

WCRP Global Sea Level Budget Group: Global sea-level budget 1993-present, 2018, Earth Syst. Sci. Data, 10, 1551-1590, https://doi.org/10.5194/essd-10-1551-2018.

Wells D.E., E.J. Krakiwsky, 1971, The method of Least Squares, Department of Geodesy and Geomatics Engineering, University of New Brunswick http://www2.unb.ca/gge/Pubs/LN18.pdf. 